\title{
PHOTON FLUX AMPLIFICATION FOR ENHANCING PHOTONIC LASER PROPULSIVE FORCES
}

\author{
P.A. Gray \\ ICRC \\ Huntsville, AL 35816 \\ M.R. Carruth, Jr. \\ D.L. Edwards* \\ ED31 Environmental Effects Group \\ Marshall Space Flight Center, AL 35812
}

\begin{abstract}
$\underline{\text { ABSTRACT }}$
An enhancement to the available force from a solar/laser sail is being investigated. This enhancement involves the use of a high power laser as the main source of propulsion or as a supplement to a solar sail. The enhancement utilizes a high power laser and multiple photon reflections to amplify the laser photon flux impinging on a sail. It is thus possible to amplify the force by as much as a factor of 50 or more. This paper explores the use of a stable optical cavity and will illustrate the optics involved in producing a stable cavity. A breadboard of the optical system was constructed and a stable cavity was demonstrated. Once the breadboard system was complete and a stable cavity achieved, the system was placed in vacuum and photon force amplification was measured using a vacuum compatible microbalance.
\end{abstract}

\section{INTRODUCTION}

The use of photons to provide propulsion has been considered. Scenarios utilizing solar sails and laser impingement have been presented. The sun provides a constant source of photons but it is not very intense. Lasers can provide greater photon flux but the technology is difficult and optimizing the use of these photons is desired. This paper presents a method to enhance the force provided by available photons. One way is to supplement the solar photons with another source of photons such as a laser.

* Member, AIAA

Copyright (C) 2001 by the American Institute of Aeronautics and Astronautics, Inc. No copyright is asserted in the United States under Title 17, U. S. Code. The U. S. Government has a royalty-free license to exercise all rights under the copyright claimed herein for governmental purposes. All other rights are reserved by the copyright owner.
Another concept involves combining a photon propelled spacecraft with a stable optical cavity ${ }^{1}$.The cavity would create multiple reflections between a stationary mirror and the spacecraft. The multiple reflections repeatedly utilize the same photons hitting the craft thus increasing the force on the craft. The stationary mirror reverses the direction of a photon reflected off the mobile mirror sending it back to the mobile mirror to exchange more of its momentum. As distance between the stationary mirror increases the complexity of maintaining a stable optical cavity increases. Therefore, it may be necessary to use this as an initial boost stage. As previously reported, a vacuum compatible microbalance was used to measure the photon force produced by an X25 solar simulator ${ }^{2}$. In this paper, the same technique was used to measure the amplified photon force resulting from stable optical cavity in conjunction with a continuous wave (CW) laser. This paper is meant to be a first look at this technique of force amplification. Future work will explore this technique in more detail.

\section{THEORY}

The concept of photon pressure is based on photons having momentum that can be transferred to an object. From Planck's law the energy, E, of a photon is:

$$
\mathrm{E}=\mathrm{h} \nu \text {, }
$$

where $h$ is Planck's constant and $v$ is the frequency of the photon. In order to make use of this equation we must relate the energy to the momentum. This can be done using equation (2) from special relativity theory:

$$
E^{2}=m_{0}^{2} c^{4}+p^{2} c^{2}
$$

where $p$ is the momentum of the particle, $m_{0}$ is the rest mass of the particle, and $c$ is the speed of light in

1 
vacuum. Since a photon has zero rest mass, equation (2) reduces to equation (3):

$$
\mathrm{E}=\mathrm{pc} \text { or } \mathrm{p}=\mathrm{E} / \mathrm{c} .
$$

Therefore, photons of higher frequency will have higher momentum than photons of lower frequency. For a solar sail, the total sum of the energies $\left(E_{T}\right)$ from all photons of all energies is the important quantity for determining the total momentum pressure for all photons hitting a sail.

Momentum for a moving object is also defined as:

$$
\mathrm{p}=\mathrm{Ft}
$$

where $\mathrm{F}$ is the force applied over time, $\mathrm{t}$.

Substituting equation (3) in equation (4) gives:

$$
\mathrm{F}=\mathrm{E}_{\mathrm{T}} / \mathrm{ct} \text {. }
$$

The total power of the photons will be defined as:

$$
\mathrm{I}=\mathrm{E}_{\mathrm{T}} \mathrm{T} \text {. }
$$

Since the force can be related to the energy, the momentum variations due to photons of different frequencies doesn't have to be considered. All that is required is that the total photon energy at the surface be known. Therefore, the photon force, $F$ in Newtons is given by:

$$
\mathrm{F}=\mathrm{I} / \mathrm{c},
$$

where I is the total power from the laser in Watts.

For a reflective surface, there is also a momentum contribution due to the reflected photons. A general equation for the photon force on a reflective surface such as a solar sail is given by:

$$
F_{\text {calc }}=(1+R)(\mathrm{L} / \mathrm{c}),
$$

where $\mathrm{R}$ is the reflectance of the sail material.

Now consider multiple photon reflections onto the sail. The result is that $F_{\text {Iotal }}$ doubles with each reflection off the second mirror (spacecraft). However, the losses increase with the mirror reflectance squared since each reflection cycle requires two reflections to complete. A general equation for total force that takes into account the loses dues to multiple mirror reflections is:

$$
\mathrm{F}_{\text {calc }}=(\mathrm{I} / \mathrm{c})(1+\mathrm{R}) \Sigma\left(\mathrm{R}^{2}\right)^{(\mathrm{N} \cdot 1)},
$$

Where $\mathrm{N}=$ number of reflection cycles.
A graph of this equation for three different mirror reflectances is found in Figure 1.

In order for equation (9) to be valid, the cavity must be stable. The relationship for a stable cavity is ${ }^{3}$ :

$$
(1-d / 2 f)^{2}<=1,
$$

where $d$ is the distance between the two mirrors and $f$ is the focal length of the mirrors. The assumption is made that both mirrors have the same focal length for this particular argument.

This stability criterion limits the distance between the two mirrors. The separation distance must be less than or equal to 4 times the mirror focal length. The stability criteria is then:

$$
\mathrm{d}<=4 \mathrm{f} .
$$

Figure 2 shows a schematic of the optical cavity.

\section{EXPERIMENTAL APPARATUS}

The test chamber for this experiment consists of a 6 way vacuum cross with 10 inch Conflat $\left.{ }^{(}\right)$flanges. The test chamber is mounted on a $600 \mathrm{l} / \mathrm{s}$ ion pump. A picture of this vacuum system is shown in Figure 3.

The experimental setup consists of an optical breadboard of the cavity. This consists of two $100 \mathrm{~mm}$ focal length (FL) concave mirrors. One of the mirrors (M1) has a small hole drilled in the center for the laser beam to pass through. The mirrors are mounted horizontally facing each other one above the other as shown in the schematic in Figure 4. The mirror with the hole in it (M1) is placed at the bottom of the cavity. The top mirror (M2) is suspended directly above the mirror with the hole (M1). The laser beam will enter the cavity by reflecting off a $45^{\circ}$ dielectric mirror. Figure 5 is a photo of the breadboard system. The laser used for breadboarding the experiment is a $35 \mathrm{~mW} \mathrm{HeNe}$. The breadboard success criterion is that the cavity is stable. A stable cavity is defined as no photons leaving the cavity as a result of reflection. Since reflectance of the mirrors is not $100 \%$, energy is lost upon reflection in the form of heat. Stability was verified by observing the spots of the HeNe laser beam on the surface of the mirrors. The issue of scattering is being neglected in this case. There is also the issue of light leaving the cavity through the center hole in the lower mirror. The top and bottom mirrors are tilted slightly to keep the laser beam in the cavity without allowing it to exit through the entrance aperture in the lower mirror. 


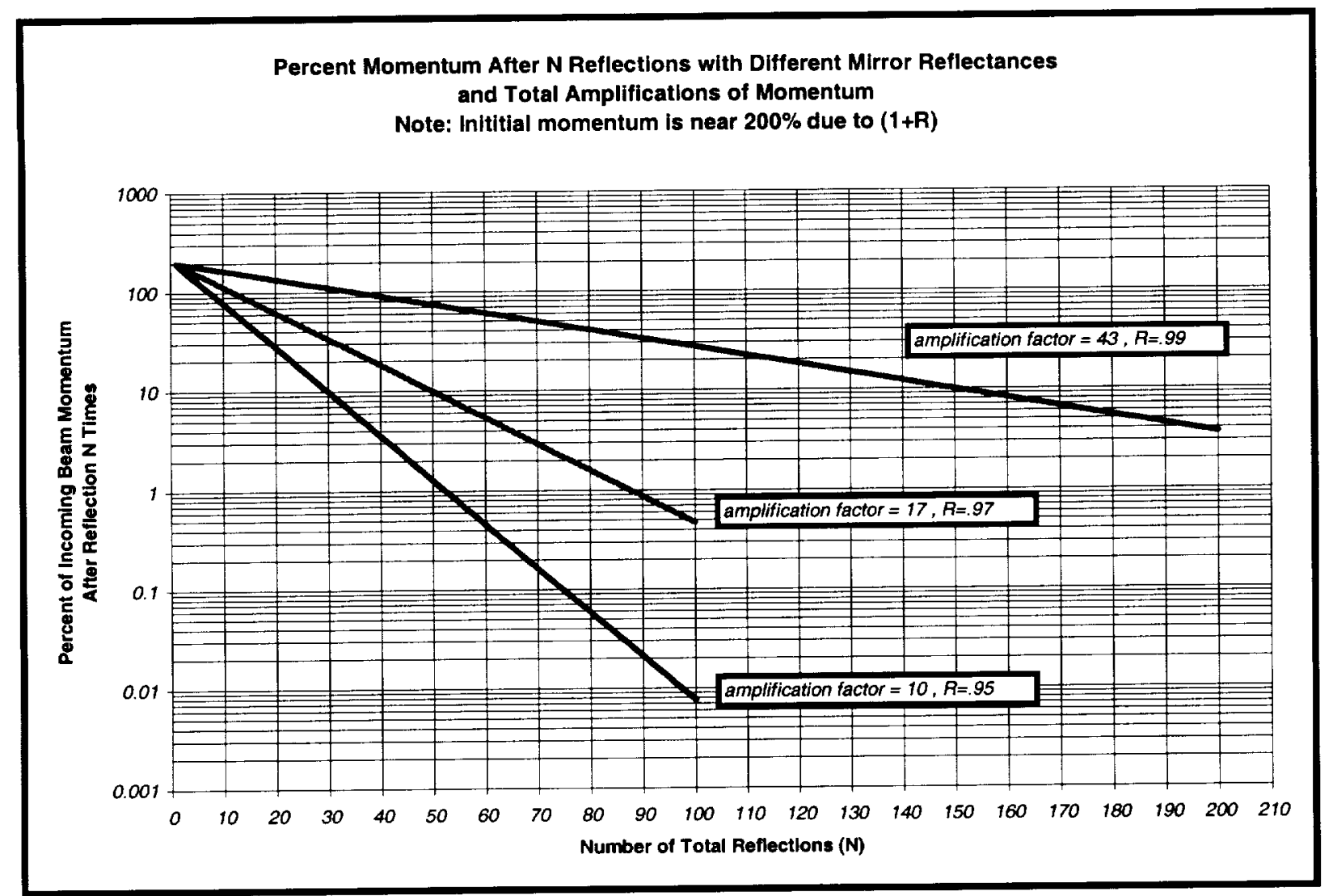

Figure 1 Force amplification possible using different mirror reflectances

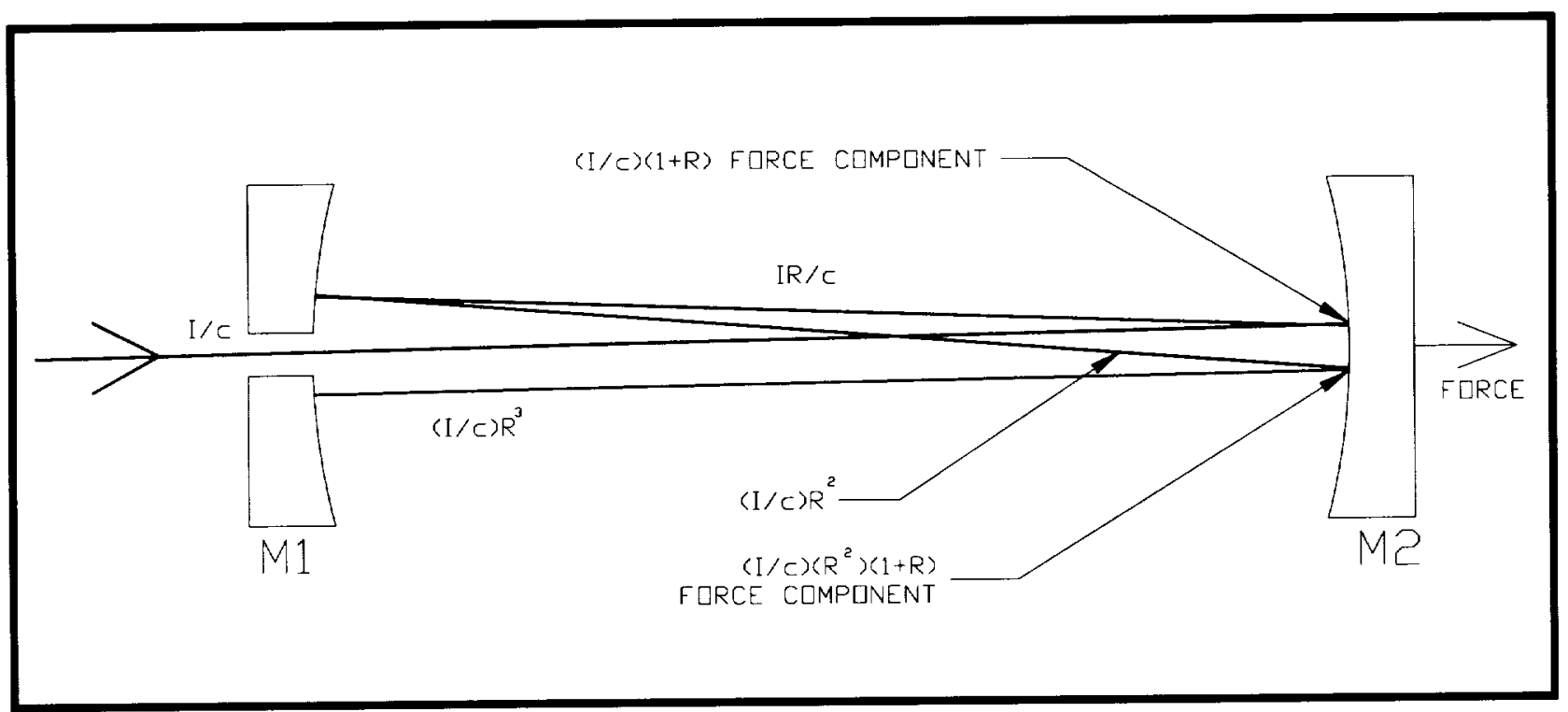

Figure 2 Schematic of optical cavity showing force components 


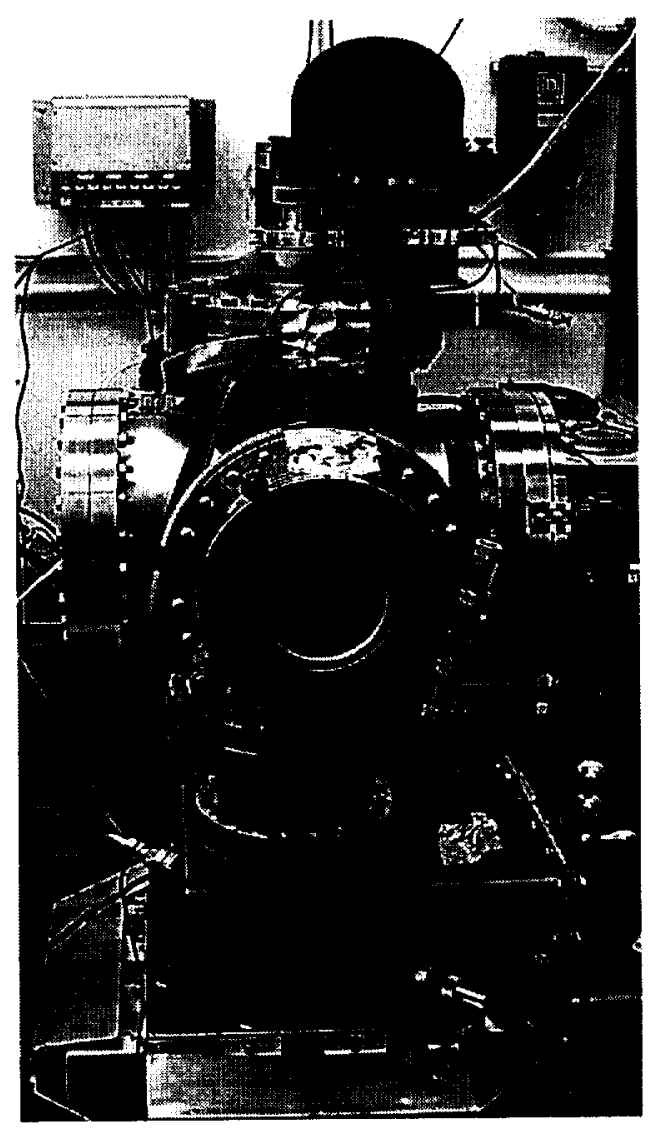

Figure 3 Vacuum system

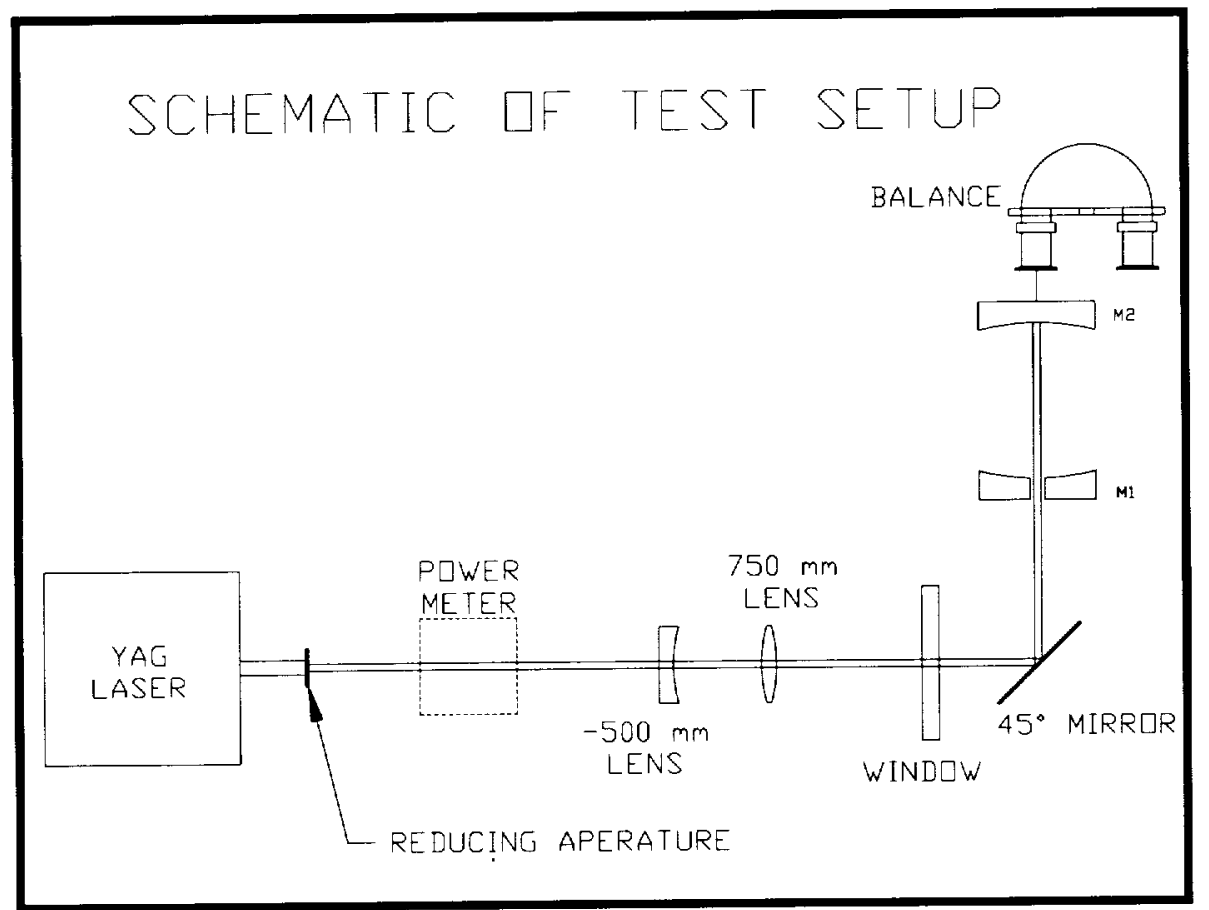

Figure 4 Schematic of test setup 


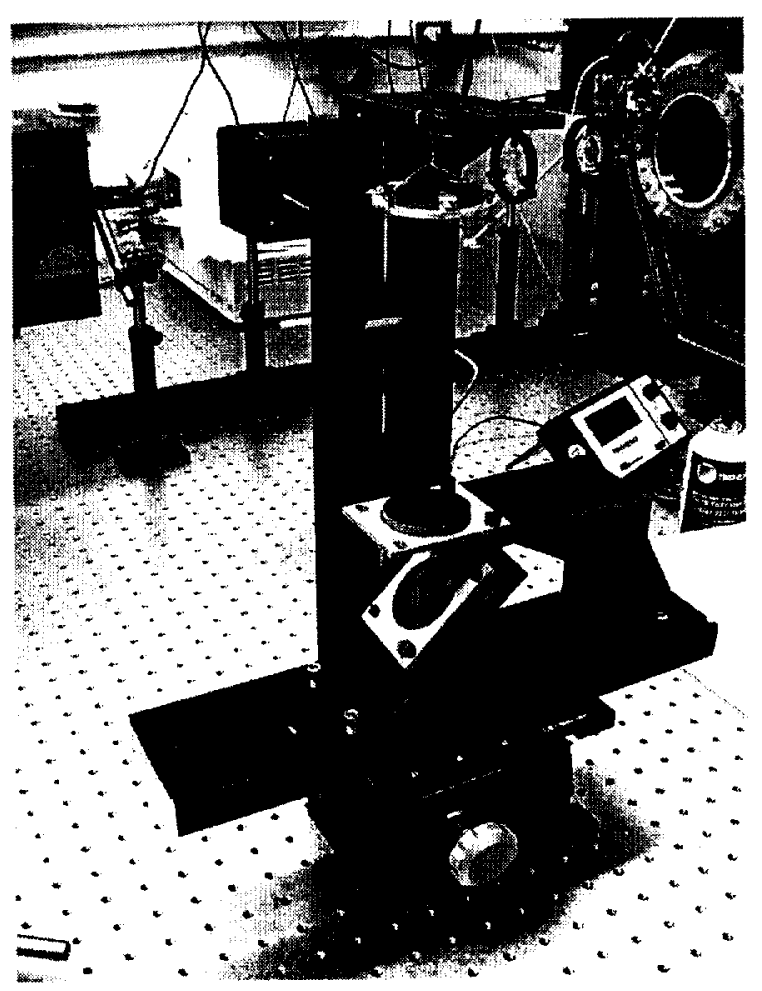

Figure 5 Optical breadboard

Tilting both mirrors has the same effect as the laser beam entering the cavity at an angle but is less complex to configure. Some simple ray trace calculations were made to show that the cavity was stable when the beam entered the cavity at a slight angle.

Once the breadboard stage was completed, the assembly was placed in vacuum in order to make actual force measurement using the high power $\mathrm{CW}$ YAG laser. Figure 6 is a photo showing the system installed in the vacuum chamber. The same $\mathrm{HeNe}$ laser was aligned so that it was coincident with the invisible YAG laser beam, which allowed for alignment of the cavity using a visible low power laser. As a comparison to the two-mirror system which provided an amplified photon force, the system was also run with the lower mirror removed. In this case incoming photons impinged on the mirror only one time.

\section{EXPERIMENTAL RESULTS}

Initial results during the breadboarding stage indicated that the laser beam would reflect off the top mirror and pass back through the hole in the bottom mirror when the laser beam entered along the cavity axis. When the top mirror was tilted and the bottom mirror was perpendicular to the beam two spots were evident on the top mirror and one spot on the bottom mirror. The beam exited the cavity through the hole in the bottom mirror thus limiting the amplification to two bounces off the top mirror before exiting the cavity. When the bottom mirror was also tilted, a series of laser spots in a ring pattern could be viewed on the top and bottom mirrors. Figure 7 shows this ring pattern for the top mirror. It is obvious that the series of spots indicate multiple reflections inside the cavity.

After it was determined that a stable laser cavity was possible with the two $100 \mathrm{~mm}$ FL mirrors, actual force measurements were made in vacuum using the $300 \mathrm{~W}$ Nd YAG CW laser. The top mirror was mounted on an adjustable base that was suspended using a wire attached to the microbalance. The lower mirror was movable but not in precise increments. When the mirrors were located inside the chamber only the top mirror surface was viewable. The first experiment used a lens to focus the YAG laser through the hole in the mirror. This worked fine for getting the beam inside the cavity but since the focal length of the lens was rather large as a result of being external to the chamber, the beam spot was still relatively focused on the top mirror. This caused the top mirror to burn and fail before any force measurements could be made. 


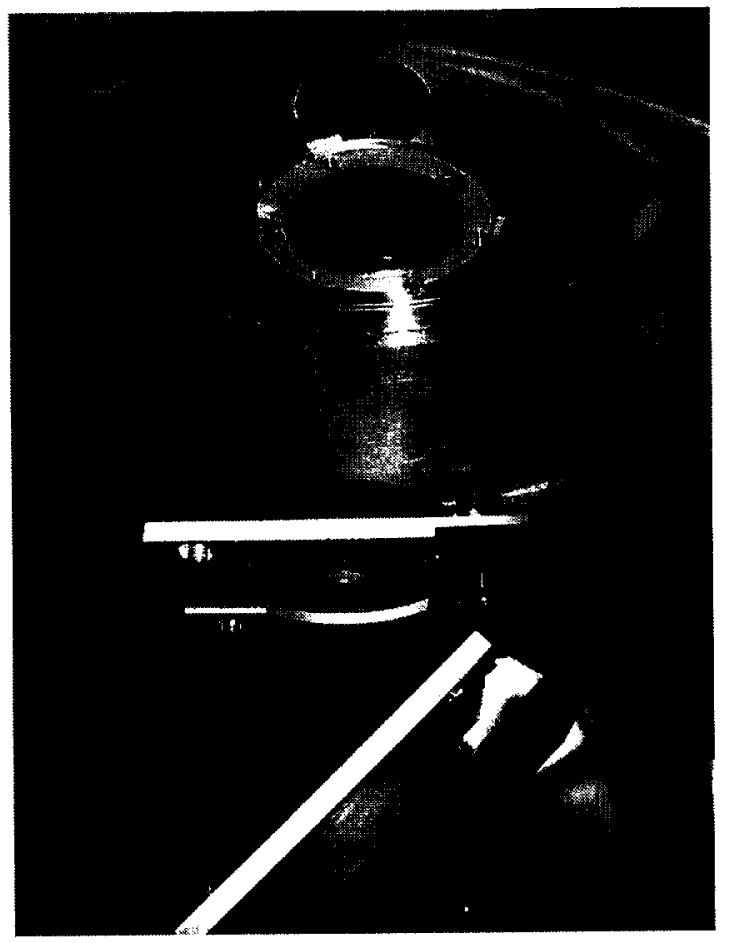

Figure 6 Optical system installed in chamber.

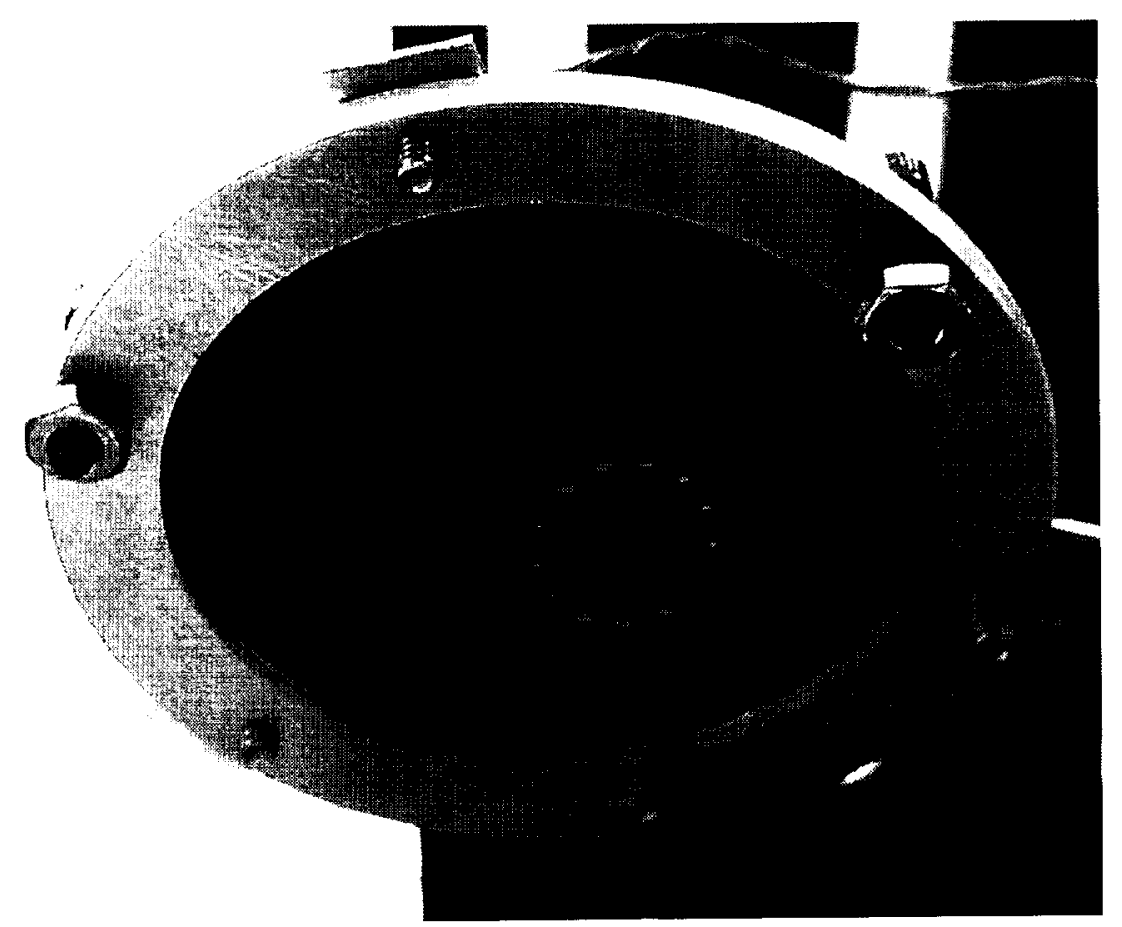

Figure 7 Multiple reflections on top mirror 
The second approach used an aperture to reduce the beam diameter of the laser to approximately $9.5 \mathrm{~mm}$ diameter from $19 \mathrm{~mm}$. This allowed the beam to pass through the bottom mirror that had a $9.5 \mathrm{~mm}$ diameter hole in it. A $-500 \mathrm{~mm}$ and $750 \mathrm{~mm}$ lens were used in series to adjust the beam diameter slightly and also provide some beam steering so that the beam could be guided through the $9.5 \mathrm{~mm}$ diameter hole. Figure 8 is a photo of the optical setup. This method did not harm the top mirror but the first mirror focused the beam on to the second mirror causing it to burn. This severely limited the amplification of photons within the cavity. Since alignment was difficult inside the vacuum chamber and space was limited, it is not known at this time if the low amplification number was due to the mirror failure or the beam leaving the cavity through the $9.5 \mathrm{~mm}$ diameter hole in the lower mirror.

For the purposes of comparison the geometry was retained but the bottom mirror was removed. The laser power was increased to produce approximately the same force without the aid of the amplification. One data set at this power level was obtained before the top mirror burned. This number was $89 \%$ of the calculated force, which was based on power measurements and system losses. Prior studies have shown measured and calculated photon force to be within a few percent. It is a possibility that, the mirror degraded prior to failing resulting in the large disparity between calculated and measured force.

In order for forces $\left(F_{m}\right.$ and $\left.F_{\text {calc }}\right)$ taken at different power levels to be compared to each other they must first be ratioed to the laser power to yield the ratios:

$$
\begin{aligned}
& \mathrm{FP}_{\mathrm{m}}=\mathrm{F}_{\mathrm{m}} / \text { Power and } \\
& \mathrm{FP}_{\text {calc }}=\mathrm{F}_{\text {calc }} / \text { Power. }
\end{aligned}
$$

In order to avoid confusion these ratios will be further defined as amplified:

$$
\left(\mathrm{FP}_{\mathrm{m}}\right)_{\mathrm{A}},\left(\mathrm{FP}_{\mathrm{calc}}\right)_{\mathrm{A}}
$$

and non-amplified:

$$
\left(\mathrm{FP}_{\mathrm{m}}\right)_{\mathrm{NA}},\left(\mathrm{FP}_{\mathrm{calc}}\right)_{\mathrm{NA}}
$$

Amplified ratios are for the case of a stable cavity with both mirrors installed and non-amplified ratios are for the case where just the top mirror was used.
Since the measured force for the case of the top mirror only is in error compared to the calculated value, the amplification ratio $\mathrm{F}_{\mathrm{m}} / \mathrm{F}_{\text {calc }}$ of 2.64 is more accurate than the amplification ratio, $\left(\mathrm{FP}_{\mathrm{m}}\right)_{\mathrm{A}} /\left(\mathrm{FP}_{\mathrm{m}}\right)_{\mathrm{NA}}$, of 3 based totally on the power normalized $\mathrm{FP}_{\mathrm{m}}$ ratios. Both of these ratios are based on the average of the two runs shown in the experiment data of Table 1.

\section{SUMMARY}

This paper has shown that photon reflection within a stable cavity does provide a means of optimizing laser photons for the purpose providing a thrust. An amplification factor of 2.64 was achieved. The theoretical number for this amplification was not achieved due to time and equipment constraints but the concept has been demonstrated. In future experiments, a larger vacuum chamber and better dielectric mirrors should improve results considerably approaching the predicted numbers shown in Fig 1.

\section{REFERENCES}

1. Thomas R. Meyer, Christopher P. McKay, Wayne R. Pryor, and Paul M. McKenna, The Laser Elevator: Momentum Transfer Using an Optical Resonator, 38th IAF Conference, Brighton, UK, October 11-17, 1987.

2. P. A. Gray, D.L. Edwards, M. R. Carruth, Jr., Preliminary Photon Pressure Measurements Using a Solar Simulator, AIAA Conference, Reno Nevada, January 8-11, 2001, \#2001-1136.

3. Amnon Yarin, Introduction to Optical Electronics, $2^{\text {nd }}$ addition. Holt, Rinehart, Winston. NY, 1976.

\section{ACKNOWLEDGMENTS}

The authors gratefully acknowledge the support of John Cole in the Advanced Space Transportation Program (ASTP) office. 


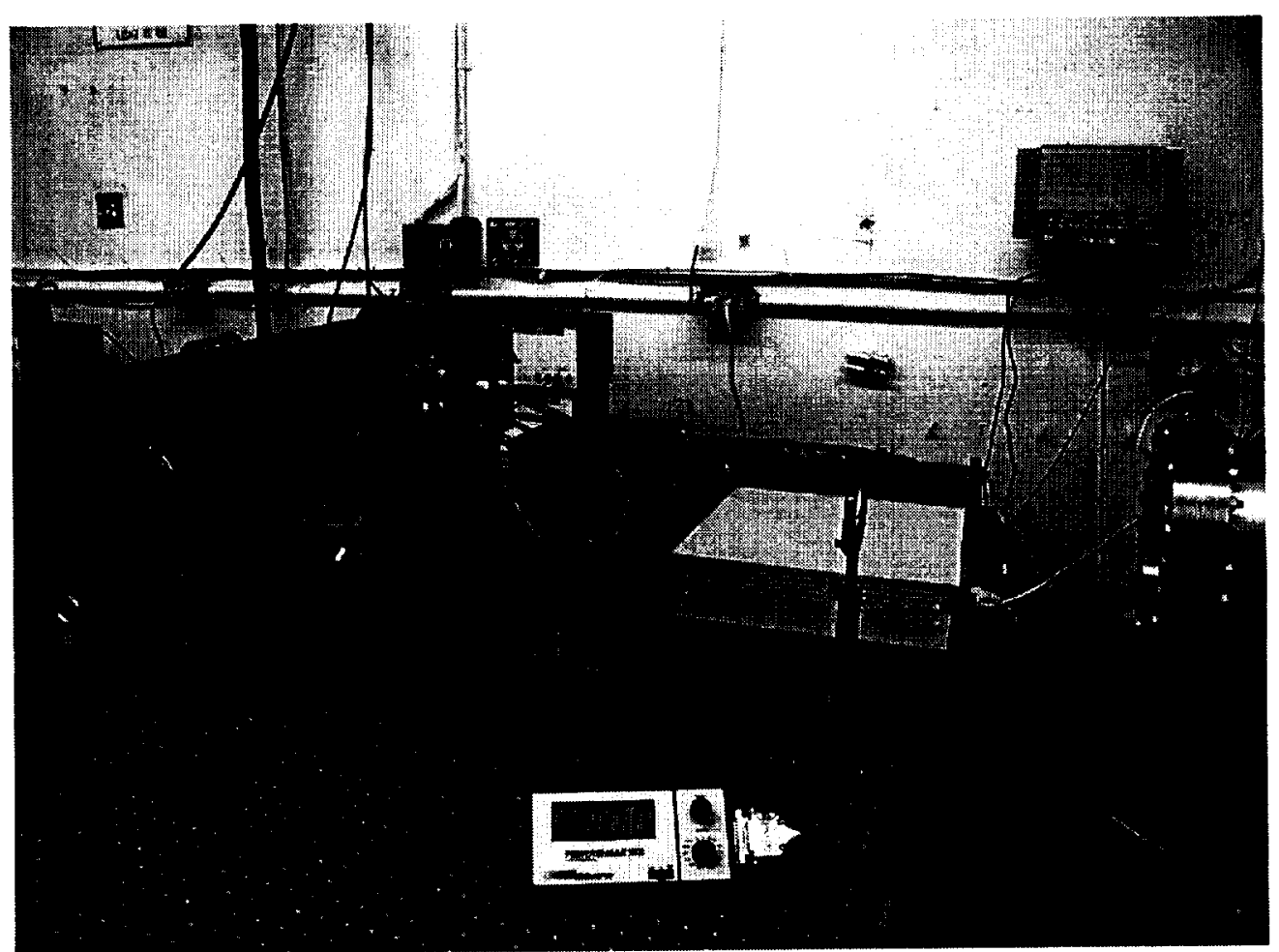

Figure 8 Photo of optical setup

Table 1 Experiment data

\begin{tabular}{|c|c|c|c|c|}
\hline Amplification & $\begin{array}{c}\text { Power } \\
\text { (Watts })\end{array}$ & $\mathrm{F}_{\mathrm{m}}(\mathrm{uN})$ & ${ }^{*} \mathrm{~F}_{\text {calc }}(\mathrm{uN})$ & $\mathrm{F}_{\mathrm{m}} / \mathrm{F}_{\text {calc }}$ \\
\hline Yes & 28.45 & 0.428 & 0.161 & 2.66 \\
\hline Yes & 23.40 & 0.344 & 0.132 & 2.61 \\
\hline$* *$ No & 88.00 & 0.439 & 0.496 & 0.89 \\
\hline
\end{tabular}

* Calculated assuming no amplification ** Top Mirror only 